Canadian Oncology

Nursing Journal

Revue canadienne

de soins infirmiers

en oncologie

Volume 30, Issue 4 • Fall 2020

elSSN: 2368-8076 


\title{
Étude qualitative descriptive sur l'expérience des patients avec réactions cutanées induites par la radiothérapie pour un cancer de la tête et du cou
}

\author{
par Maurene McQuestion et Angela Cashell
}

RÉSUMÉ

La majorité des patients recevant de la radiothérapie auront une réaction cutanée radio-induite allant d'un érythème léger à une desquamation humide étendue. Les réactions cutanées attendues varieront en fonction du diagnostic de cancer, de la dose et de la taille du champ d'irradiation ainsi que d'autres facteurs liés au traitement et au patient. Récemment, la recherche a dissipé les mythes longtemps entretenus sur les produits pour le soin de la peau qui auraient été à éviter avant le traitement, tout comme le déodorant et l'antisudorifique durant les traitements contre le cancer du sein (on craignait une surdose accrue pour la peau causée par l'utilisation de ces produits). Cette limitation a été levée. Or, la plupart des recherches sur les réactions cutanées radio-induites ont été menées auprès de femmes atteintes d'un cancer du sein; aucune n'a été trouvée sur les patients atteints de cancer de la tête et du cou, alors que de tous les groupes de patients, ce sont très souvent ceux-ci qui présentent les réactions cutanées les plus graves. Nous avons fait appel à la méthode descriptive interprétative qualitative basée sur le travail méthodologique de Thorne (1997) pour interroger neuf participants sur leurs réactions cutanées par suite de radiations pour leur cancer de la tête et du cou. Trois thèmes sont ressortis des entretiens : 1) l'expérience des symptômes; 2) la comparaison avec d'autres; 3) l'expérience du soutien et de l'information. Les implications comprennent des recommandations pour la pratique et l'éducation des patients.

\section{INTRODUCTION ET HISTORIQUE}

$\mathrm{L}$ a majorité des patients recevant une radiothérapie auront ـune réaction cutanée, allant de l'érythème léger à une desquamation humide grave. Les réactions cutanées attendues varieront en fonction du diagnostic de cancer, de la dose totale et du programme de fractionnement des rayonnements, de la taille du champ d'irradiation ainsi que d'autres facteurs liés au traitement et au patient (ex. âge, sexe, mauvais état nutritionnel) (Glover et Harmer, 2014; Singh, 2016). L'altération

\section{AUTEURES}

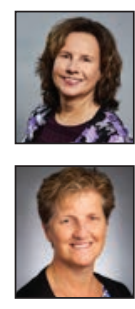

Maurene McQuestion, B.A., B.Sc.inf., M.Sc., CSIO(C)

Auteure-ressource : stampersmokey@yahoo.ca

Angela Cashell, TRM, M.Sc. physique de la peau peut causer de la douleur, des démangeaisons ou une sensation de brûlure, et ainsi nuire aux activités de la vie quotidienne, au sommeil, au port de certains vêtements, aux mouvements et à l'activité physique, de même qu'à l'humeur et à l'image corporelle, à l'autonomie, aux soins personnels et à la qualité de vie (Feight et al., 2011; Schur et al., 2011). La plupart des recherches se concentrent sur la comparaison et l'évaluation de l'efficacité des produits (lotions, crèmes, onguents, pansements, etc.) pour prévenir ou réduire la gravité des réactions cutanées ou les gérer, mais avec des preuves limitées pour ce qui est de l'utilisation concrète à titre préventif ou curatif (Bolderson et al., 2018; Ferreira et al., 2017) Des analyses documentaires et critiques des écrits ont été effectuées, et des lignes directrices ont été élaborées sur la prévention et la gestion de la radiodermite, pour influer sur la pratique clinique (Chan et al., 2014; McQuestion 2011; Salvo et al., 2010; Wong et al., 2013). L'ensemble de la recherche quantitative a été confronté à de nombreux défis méthodologiques, notamment la petite taille des échantillons, le manque d'uniformité terminologique pour définir le stade des réactions cutanées, la variation des outils de mesure et la diversité des résultats évalués.

Récemment, la recherche a dissipé des mythes longtemps entretenus sur l'évitement des produits de soins de la peau pendant une période déterminée avant le traitement, l'utilisation de déodorant ou d'antisudorifique durant le traitement du cancer du sein, et le risque d'une surdose causée par ces produits. Les patients ne sont plus incités à cesser temporairement d'appliquer tout déodorant ou antisudorifique, et d'employer des lotions ou crèmes avant le traitement (Morley et al., 2014; Tse et al., 2016). Malgré tout, les milieux ont été lents à adopter ces changements fondés sur des preuves (comme le lavage avec un savon doux, l'utilisation de déodorants ou d'antisudorifique, l'application de lotions ou de crèmes avant le traitement, etc.). Des incohérences dans la pratique persistent d'un clinicien et d'un milieu de pratique à un autre, ce qui entraîne un manque d'uniformité dans l'information et le soutien fournis aux patients subissant de la radiothérapie (Bolderston et al., 2018). Il en découle toutes sortes d'expériences pour les patients.

Seulement deux études qualitatives ont été repérées sur l'expérience des patients avec une toxicité cutanée aiguë due à la radiothérapie; les deux pour des femmes atteintes d'un cancer du sein (Andersen et al., 2018, 2018; Schnur et al., 2011). Aucune recherche n'a été trouvée sur l'expérience d'une réaction cutanée aux radiations chez les patients atteints d'un 
cancer de la tête et du cou spécifiquement. Dans ce groupe de patients, la radiodermite est l'un des effets secondaires les plus courants : jusqu'à $80 \%$ des patients souffriront d'une dermatite modérée à grave. Cet effet secondaire résulte de l'administration de doses de traitement élevées (56 à $70 \mathrm{~Gy}$ ) au site primaire et aux ganglions cervicaux unilatéraux ou bilatéraux, et du fait que le site primaire ou la cible de rayonnement visée se trouve à proximité immédiate de la peau. Des réactions modérées à graves se produisent malgré l'utilisation de la radiothérapie à modulation d'intensité (IMRT) comme norme de soins dans ce groupe de patients, une approche qui vise à améliorer la distribution des doses (Singh et al., 2016).

Jusqu'à présent, la recherche ne s'est pas attardée à comprendre l'expérience des patients, l'impact de la réaction cutanée sur la façon dont ils font face au traitement, l'accès à l'information et aux ressources, ou la façon dont la réaction cutanée est gérée du point de vue des patients. Les recommandations actuelles en matière de soins sont souvent basées sur la vision et l'expérience du clinicien et fondées sur des faits dont la qualité varie (Bolderston et al., 2018). Bien que des problèmes psychosociaux rencontrés par les patients aient été signalés, la vision complète des patients sur les réactions cutanées n'a pas été décrite (Campbell et Illingworth, 1992; Freight 2011). Sutherland et collaborateurs (2017) ont évalué le stress psychologique dans un sous-ensemble de femmes atteintes d'un cancer du sein qui participaient à un essai contrôlé randomisé sur la gravité des réactions cutanées aiguës; ils ont constaté que les patients stressés étaient deux fois plus susceptibles de présenter une réaction cutanée grave et cinq fois plus susceptibles de présenter une desquamation humide. Comprendre l'expérience des patients devrait mener à de meilleures interventions axées sur le patient, à un meilleur soutien, à une meilleure documentation, et à des recommandations de produits permettant de mieux gérer la réaction cutanée.

\section{BUT DE L'ÉTUDE}

Cette étude qualitative a été menée pour mieux comprendre comment les patients atteints d'un cancer de la tête et du cou abordent les réactions cutanées radio-induites, comment ces réactions affectent la manière dont ils vivent le traitement, quel est leur accès à l'information et aux ressources, et comment les réactions cutanées sont gérées de leur point de vue.

Jusqu'à présent, très peu d'attention a été accordée à l'expérience d'une réaction cutanée aux radiations du point de vue des patients qui vivent avec l'impact du diagnostic et $\mathrm{du}$ traitement. Létude actuelle comblera une lacune dans la littérature et sensibilisera le personnel clinique, notamment les infirmières autorisées, les radiothérapeutes et les radio-oncologues qui s'occupent des patients traités pour un cancer de la tête et du cou. Il est également important d'ajouter aux connaissances dont les infirmières et les autres cliniciens ont besoin pour éclairer leur pratique, de même qu'aux recherches fondées sur des données probantes qui ont orienté la gestion des soins aux patients et l'éducation en matière de dermatite radique chez les patients atteints d'un cancer de la tête et du cou.

\section{MÉTHODOLOGIE}

La question de recherche était : « Du point de vue du patient, comment est vécue une réaction cutanée due aux radiations? » Les participants qui ont vécu le phénomène ont été interrogés de 2 à 3 mois après leur traitement. La participation n'était pas possible plus tôt en raison de la douleur liée à la mucosité buccale, de la xérostomie (sécheresse) et de l'odynophagie (douleur à la déglutition), qui rendent particulièrement difficile le dialogue verbal lors d'un entretien.

Échantillon/contexte. Les patients ont été recrutés au cours de leur dernière semaine de radiothérapie à l'unité de traitement ou lors de leur dernier rendez-vous hebdomadaire à la clinique de suivi. Lorsqu'une infirmière autorisée ou un radiothérapeute identifiait un patient qui avait une réaction cutanée aux radiations à la fin du traitement, il l'informait sur l'étude en lui remettant une lettre d'introduction qui en expliquait le but. Si le patient était intéressé, un membre de l'équipe de recherche (sans aucun lien clinique avec le participant potentiel) le rencontrait pour répondre à ses questions et obtenir son consentement en lui faisant signer un formulaire qui détaillait l'étude. Le patient était ensuite contacté après 4 à 6 semaines pour fixer un entretien environ 2 à 3 mois après la fin du traitement, en conjonction avec un rendez-vous de suivi de routine afin de ne pas obliger de visite supplémentaire au centre de cancérologie.

Un échantillonnage ciblé a été utilisé afin d'avoir des participants présentant des variations démographiques (hommes, femmes, plus jeunes ou plus âgés, diagnostic), mais dont l'expérience et les perspectives auraient des points communs avec les autres. Cela a permis d'obtenir un échantillon homogène de patients présentant des réactions cutanées aux radiations et, en même temps, un échantillon suffisamment hétérogène pour maximiser les informations et la variation des thèmes qui se sont dégagés, et donc assurer la saturation et l'adéquation des données (Morse, 2015; Tong, Sainsbury et Craig, 2007). Les critères d'inclusion étaient les suivants : 1) participants de 18 ans ou plus ayant vécu le phénomène recherché (réaction cutanée aux radiations); 2) capables de lire et de communiquer verbalement en anglais; 3) ayant reçu des radiations à la tête et au cou; 4) désireux de participer à l'étude. Certains critères d'exclusion ont été appliqués : 1) récidive de cancer; 2) traitement antérieur par radiation pour un autre type de cancer; 3) traitement par radiation à des fins palliatives; 4) soins cliniques directs reçus de l'un ou l'autre des chercheurs.

Déroulement de l'étude. Les entretiens ont été menés à l'hôpital dans une salle de conférence de la clinique externe ou dans l'unité d'hospitalisation des patients admis pour une chimiothérapie. Les participants ont été encouragés à raconter leur expérience de réaction cutanée aux radiations. La question initiale était la suivante : « Comment avez-vous vécu les réactions cutanées aux radiations? » Un guide d'entretien semi-structuré permettait d'élaborer sur les autres sujets suscités par la discussion (Gillan et al., 2014). Les questions portaient également sur ce que les patients s'étaient fait dire concernant les changements cutanés à prévoir au cours du 
traitement, la manière de soigner les réactions, l'impact de la réaction cutanée sur leur vie quotidienne et ce qu'ils conseilleraient aux autres patients à ce sujet. Les entretiens ont duré entre 30 et 60 minutes par patient.

Le recrutement s'est avéré plus difficile que prévu (voir « Limites » ci-après). Aucun nouveau thème n'est apparu après neuf entretiens. Il a donc été décidé de clore l'étude à ce stade. Les neuf entretiens effectués ont été analysés et les résultats sont présentés ci-dessous.

Analyse des données. Tous les entretiens ont été enregistrés et transcrits textuellement. Le logiciel Nvivo a été utilisé pour la gestion des données. Les descriptions complètes de l'expérience des participants concernant leurs réactions cutanées ont été lues pour avoir une idée de l'ensemble. Le contenu a d'abord été analysé dans le contexte de l'entretien individuel, puis les données été reliées entre elles, avec les autres entretiens. L'analyse s'est concentrée sur les riches descriptions transcrites à partir des entretiens avec les patients et sur l'identification des thèmes grâce à une analyse poussée des données et à la compréhension des perspectives des participants, ainsi que des relations et des interprétations de l'expérience afin de rapporter les données d'une manière significative et utile pour la pratique clinique (Thorne, 1997; Thorne, et al., 2016; Thorne, 2018).

\section{RÉSULTATS}

Vingt et un patients ont été approchés dans la zone clinique par les radiothérapeutes ou les infirmières spécialisées en oncologie. Quatorze ont accepté d'être contactés par un des chercheurs pour qu'on leur explique l'étude et ont consenti à être interviewés. Trois d'entre eux n'ont pas pu être joints pour fixer une date d'entretien et un autre s'est retiré de l'étude parce que le moment choisi pour l'entretien ne convenait pas. Dix personnes ont donc été interviewées, mais un entretien n'a pas été inclus dans l'analyse en raison de difficultés techniques liées à l'enregistrement.

Données démographiques. Le tableau 1 rassemble les données démographiques. Au total, 7 hommes et 2 femmes, âgés de 50 à 79 ans, ont été interrogés. Parmi les participants, 7 étaient de type caucasien et 2 étaient d'origine chinoise. Le diagnostic comprenait des carcinomes épidermoïdes de l'oropharynx (base de la langue) et de l'hypopharynx, de la glotte, de la glande parotide et du nasopharynx. La majorité (8) a reçu quotidiennement $70 \mathrm{~Gy} / 35$ \# de radiations primaires pour traiter un stade avancé de la maladie. Deux participants recevaient de la chimiothérapie en même temps. Six des 9 ont présenté une desquamation humide confluente. Trois ont eu une desquamation sèche étendue ou une desquamation humide irrégulière. La répartition par âge reflétait la répartition des patients atteints d'un cancer de la tête et du cou qui sont vus au centre de cancérologie. Tous étaient mariés et avaient fait des études secondaires ou collégiales/universitaires.

\section{Données d'entrevues-Thèmes}

Les résultats sont présentés sous forme de thèmes cernés à partir des données des participants. Trois thèmes principaux

\begin{tabular}{|l|l|l|}
\hline \multicolumn{2}{|l|}{ Tableau 1: Données démographiques des participants } \\
\hline \multirow{2}{*}{ Âge } & $50-59$ & 3 \\
\cline { 2 - 3 } & $60-69$ & 5 \\
\cline { 2 - 3 } & $70-79$ & 1 \\
\hline \multirow{2}{*}{ État matrimonial } & Marié & 9 \\
\hline \multirow{2}{*}{ Education } & Collège / Université & 5 \\
\cline { 2 - 3 } & École secondaire & 4 \\
\hline
\end{tabular}

sont ressortis des entretiens : 1) l'expérience des symptômes; 2) la comparaison avec d'autres; 3) l'expérience concernant le soutien et l'information reçus. Les thèmes sont numérotés pour la clarté de la présentation, mais ne reflètent pas une conceptualisation linéaire par les participants ou les chercheurs, ni un ordre hiérarchique.

\section{Thème 1 : L'expérience des symptômes}

Les répondants à l'étude ont étiqueté la réaction cutanée comme l'un des multiples symptômes ressentis pendant le traitement. Parmi ces multiples symptômes, ils ont nommé les douleurs buccales, la xérostomie/sécheresse buccale, la difficulté à manger et à avaler, la fatigue, la perte d'appétit, la perte de poids et d'autres préoccupations liées aux effets secondaires $\mathrm{du}$ traitement. Ils ont eu tendance à minimiser l'expérience globale de réaction cutanée par rapport aux autres symptômes qui sont apparus pendant le traitement, le voyant comme un symptôme de plus parmi les nombreux symptômes et problèmes avec lesquels ils devaient composer. Ils ont reconnu que si la réaction cutanée était gênante et s'aggravait au cours du traitement, la superposition d'autres symptômes et l'expérience globale du traitement avaient un impact plus grand sur la détresse ressentie pendant le traitement.

Pour la plupart, les réactions cutanées ont commencé par des changements après 3 à 4 semaines de traitement. Les changements de la peau du cou et du champ de traitement étaient visibles à divers degrés pendant le reste du traitement. La plupart ont présenté une desquamation humide confluente. Deux personnes n'ont pas eu de réaction avant la fin du traitement, alors que d'autres symptômes plus graves dominaient.

C'était inconfortable, mais c'était le moindre de mes pro-

blèmes avec la radiothérapie à traverser.

Je ne pense pas que ça ait été si pire pour moi; je n'ai pas eu de réaction avant la fin du traitement.

Les participants ont décrit d'autres symptômes associés aux réactions cutanées, notamment la douleur. La douleur croissante a coïncidé avec le moment où leurs réactions cutanées se sont accélérées au cours de la cinquième semaine de traitement. Plusieurs ont décrit la douleur aux semaines 6 et 7 comme « très forte », les empêchant de sortir. Lorsqu'ils n'étaient pas 
au centre de cancérologie ou en route entre leur domicile et le lieu de traitement, les participants ne sortaient pas de chez eux en raison de la gêne provoquée par les réactions cutanées, du besoin de pommades ou de pansements et de l'autogestion des autres symptômes liés au traitement. Pour ceux qui utilisaient des pommades, mais essayaient d'éviter les pansements durant le jour, ils préféraient souvent porter un t-shirt ou une camisole très échancrés pour éviter le contact des vêtements avec la peau. Laspect des réactions cutanées et la façon dont ils ont dû adapter leurs vêtements ont réduit leur envie de sortir en public pour toute autre raison que se rendre au traitement.

À mesure que le traitement avançait, les réactions cutanées s'intensifiaient. L'effet le plus important pour les patients a été la perturbation de leur sommeil. Plusieurs ne pouvaient pas dormir dans leur lit et ont dû se coucher dans une chaise longue ou s'allonger sur le canapé toute la nuit, pendant le reste du traitement et dans les semaines suivant le traitement.

J'avais du mal à dormir la nuit. Je devais m'asseoir dans mon lit. Je ne pouvais pas m'allonger sur le côté parce que ça faisait mal.

La nuit, si je ne faisais pas attention, je me réveillais chaque fois que la couverture frôlait accidentellement mon cou ou ma peau.

D'autres participants ont décrit les symptômes qu'ils ont ressentis durant la phase de guérison après le traitement, comme la sensation de tiraillement ou d'étirement de la peau du cou.

Je faisais très attention à mes mouvements, car quand on bouge et que ça commence à guérir, ça tire.

Pendant ma guérison, je restais la plupart du temps assise sur une chaise.

\section{Thème 2 : La comparaison avec d'autres}

Les participants analysaient l'état des autres patients dans la salle d'attente, la clinique de radiothérapie et les zones d'examen de la clinique, et remarquaient ceux qui présentaient des réactions cutanées à divers degrés. Certains avaient besoin de pommades et de pansements ou de couvrir leur visage et leur cou. Certains participants se comparaient pour normaliser leur expérience ou comprendre ce qui les attendait en observant les réactions cutanées des autres. Pour beaucoup, cette comparaison les aidait à mieux affirmer leur état, voyant que leur peau était comme celle des autres à la même phase du traitement, que «ça allait » et qu'ils n'étaient « pas plus mal » que les autres.

Je n'arrêtais pas de remarquer tous les autres patients qui passaient et de voir les rougeurs et les plaies et tout, et de me demander si j'allais passer par là.

J'ai remarqué d'autres patients qui avaient la même chose et leur peau était pire que la mienne.

Je ne pense pas avoir eu une mauvaise réaction cutanée par rapport à d'autres.
Thème 3 : L'expérience concernant le soutien et l'information reçus

Dans leur grande majorité, les participants ont déclaré qu'ils conseilleraient aux autres d'écouter l'équipe de soins de santé et de suivre leurs conseils et instructions. Ils se sont sentis écoutés et bien soutenus dans la gestion de leurs symptômes, y compris les réactions cutanées. Ils ont formulé leurs commentaires comme des conseils qu'ils donneraient à d'autres patients, mais ils n'avaient que peu de conseils à donner aux cliniciens. Garder une attitude positive était le plus important conseil donné pour les autres patients.

Soyez prêts. Ça s'en vient, ça va être douloureux, mais vous devez rester positif.

Veillez à mettre de la crème hydratante tous les jours.

Il suffit de s'hydrater, encore, encore et encore.

Les participants ont également souligné l'importance d'être informés au bon moment. Ils ont senti que l'équipe clinique surveillait, remarquait les changements et faisait des suggestions. Avoir de l'information à mesure que la réaction cutanée évoluait a été utile, plutôt que d'obtenir trop d'informations dès le début sur les changements à venir.

Soyez attentifs à ce qu'on vous dit.

C'était bien mieux que d'être accablée d'informations en vrac dès le début. C'était merveilleux de voir l'équipe répondre à chacun de mes besoins spécifiques au moment où il se présentait.

\section{LIMITES DE L'ÉTUDE}

L'une des limites de l'étude pourrait être le manque de participantes du sexe féminin. Bien que le ratio hommes/femmes recevant un traitement pour le cancer de la tête et du cou soit de 2:1, voire 3:1, il aurait été utile d'entendre davantage de femmes, susceptibles de vivre les choses autrement.

Le recrutement s'est avéré plus difficile que prévu. Alors que 8 à 12 patients suivent un traitement contre le cancer de la tête et du cou chaque semaine au centre de cancérologie, seuls 25 patients ont accepté d'être contactés pour recevoir plus d'informations ou ont donné leur consentement pour être contactés afin d'organiser un entretien. Le milieu clinique est très occupé et l'identification de patients potentiels pour des études de recherche non liées à un traitement peut ne pas être la priorité des cliniciens affairés. De nombreux patients approchés ne se sentaient pas assez bien pour envisager de participer à une étude et certains, qui avaient donné leur consentement, n'ont pas pu y participer par la suite. Diverses raisons ont été évoquées : le fait de ne pas se sentir pas assez bien pour participer, de demeurer loin, de ne pas arriver à coordonner un entretien avec un rendez-vous à la clinique, de ne pas vouloir multiplier les visites à l'hôpital en début de convalescence. Des entretiens téléphoniques auraient pu accroître la participation, mais de nombreux patients atteints d'un cancer de la tête et du cou ont en fait de la difficulté à parler au téléphone tout de suite après le traitement. 


\section{DISCUSSION ET IMPLICATIONS}

Cette étude est la première étude qualitative portant spécifiquement sur l'expérience des patients en cas de réaction cutanée aux radiations associée au traitement d'un cancer de la tête et du cou. D’autres études qualitatives ont été menées auprès de patients atteints d'un cancer de la tête et du cou, mais elles se sont plutôt concentrées sur l'expérience ou l'impact global du traitement par radiations et sur les symptômes ressentis pendant et après le traitement (McQuestion et Fitch, 2016; Molassiotis et Rogers, 2012; Wells, 1998, 2007).

Les réactions cutanées radio-induites subies par les participants sont l'un des nombreux effets secondaires relevés, mais ce symptôme spécifique ne suscite pas un désir de compréhension plus poussé. Les patients atteints de cancer de la tête et du cou présentent des réactions cutanées radio-induites dans le contexte de multiples autres symptômes graves et impactants : douleurs buccales, mucosites, odynophagie, altération du goût, difficultés à manger et à avaler, perte d'appétit, perte de poids, déshydratation, fatigue, etc. Haisfield-Wolfe (2012) a identifié un schéma commun de onze symptômes problématiques que les patients atteints de cancer de la tête et du cou éprouvent au cours du traitement. Même si les participants à cette étude ont eu tendance à minimiser l'expérience de la réaction cutanée dans le contexte des autres symptômes concomitants et plus débilitants, le fait de vivre au quotidien avec les réactions cutanées a quand même eu un effet important. La douleur, l'inconfort, le besoin de pommades et de pansements, les changements de routine et les perturbations du sommeil, en particulier la nuit, étaient tous majeurs. Les participants avaient le réflexe de se comparer aux autres pour savoir s'ils se débrouillaient bien. Létat de leur peau, qui nécessitait des applications répétées de lotions, de crèmes ou de pommades et des pansements, de même que les besoins personnels liés à d'autres symptômes ont incité les participants à rester chez eux et à ne se déplacer que pour leur traitement. Considérant que le traitement est quotidien pendant 4 à 7 semaines, cela pourrait créer ou aggraver le sentiment d'isolement social chez certains patients ayant moins de soutien ou de ressources sociales.

Le sommeil a également été affecté, les patients se réveillant fréquemment en raison de la douleur ou de l'inconfort causés par les réactions cutanées ou devant changer de routine, notamment en passant d'un lit à une chaise longue pour dormir. On a établi les troubles aigus et chroniques du sommeil comme l'un des cinq principaux facteurs de risque de détresse; ils peuvent entraîner une fatigue diurne, une humeur irritable et des troubles cognitifs (Van Hoose et al., 2015). Les troubles du sommeil ne devraient pas être négligés chez ces patients en tant que conséquence due aux réactions cutanées.

Des études menées sur des femmes atteintes d'un cancer du sein ont montré que les modifications de la peau causée par la radiothérapie affectent leur qualité de vie et les empêchent de fonctionner au quotidien, notamment lorsqu'il s'agit de changer de vêtements et de dormir (Schnur, 2010). Des difficultés à trouver le sommeil étaient notamment liées à la douleur et à la sensation de brûlure. Le besoin fréquent de se repositionner créait également des difficultés à rester endormi. Schnur, Ouellette, DeLorenzo, Green et Montgomery (2010) ont interrogé 20 femmes pour comprendre l'impact des réactions cutanées sur la qualité de vie et conclu que « les effets d'une toxicité cutanée aiguë vont bien au-delà de l'épiderme ».

Écouter l'équipe de traitement et suivre ses conseils a été perçu comme très important par les participants à notre étude. Ils avaient aussi quelques conseils pour les autres patients : rester positif, bien hydrater la peau et être attentif aux conseils de l'équipe de soins. Une étude sur les préférences des patients en matière de renforcement pédagogique concernant la prévention des réactions cutanées radio-induites a révélé que le renforcement verbal et vidéo était préféré aux documents écrits (Laszewski, et al., 2016). Les participants à cette étude n'ont pas indiqué un format particulier pour fournir des informations comme étant des plus importants. Néanmoins, les patients reçoivent des documents écrits, se font conseiller des vidéos, profitent d'un enseignement individuel et suivent des cours de groupe où des conseils pour gérer les réactions cutanées sont mis de l'avant. Ils ont également une évaluation quotidienne et un examen hebdomadaire accompagné de conseils, respectivement par le radiothérapeute et l'infirmière autorisée qui les soignent. Les ressources verbales et écrites peuvent constituer la norme de la pratique clinique dans la plupart des contextes, mais l'utilisation de la vidéo, du virtuel ou d'autres technologies doit aussi être considérée comme une option pour répondre aux besoins des patients.

\section{IMPLICATION POUR LA PRATIQUE ET L'ÉDUCATION}

Les patients atteints d'un cancer de la tête et du cou qui ont de la radiothérapie peuvent ne pas ressentir la gravité des réactions cutanées au cours du traitement de la même manière que d'autres patients dont le diagnostic et les traitements par radiations sont différents, en raison de la superposition de multiples symptômes ou effets secondaires autres au cours du traitement aigu par radiations et pendant la convalescence. Il est important que les cliniciens comprennent et reconnaissent que le fait de vivre au quotidien avec des réactions cutanées demeure grave, même si les patients tendent à le minimiser. L'équipe doit également reconnaître les réactions cutanées comme un symptôme dans la multitude de symptômes ressentis par les patients et comprendre l'importance de le reconnaitre et de le gérer. Les informations sur la procédure et la gestion ainsi que l'expérience d'autres patients et ce qu'ils ont trouvé utile profiteront également aux patients. Ces informations peuvent comprendre la chronologie et le schéma des symptômes de radiodermite, l'importance de s'assurer que la réaction cutanée est reconnue et soignée, les effets sur la vie quotidienne, la gestion à domicile avec les soins personnels et l'isolement social potentiel, les conséquences sur le sommeil et les stratégies à privilégier, la superposition d'autres symptômes, la façon dont les patients se comparent aux autres et l'accent mis sur l'importance de l'information et du soutien. Il peut être utile d'intégrer les expériences des patients dans les documents d'éducation des patients, parallèlement aux conseils traditionnels habituels fondés sur des données probantes. 
Léducation virtuelle a augmenté et augmentera en raison du nombre croissant de patients, de la planification en cas de pandémie et des difficultés liées aux ressources opérationnelles ou budgétaires. Toutefois, l'accès aux membres de l'équipe clinique et aux soins infirmiers continuera d'être essentiel aux interactions patients-cliniciens, à l'évaluation, et au soutien des patients présentant des réactions cutanées radio-induites dans le contexte des multiples groupes de symptômes que connaissent les patients atteints de cancer de la tête et du cou. Veiller à ce que ces interactions aient lieu peut avoir un impact sur le flux des patients, la conception des cliniques et les rôles au sein des services de radiothérapie, et devrait s'ajouter à l'éducation virtuelle des patients et au soutien des patients par des contacts réguliers ou de suivi.

La prévalence des réactions cutanées graves a également évolué au fil du temps. La pratique a changé pour refléter une plus grande concentration sur l'intervention précoce, l'utilisation de pansements spéciaux qui peuvent rester en place pendant le traitement, de même que la réduction des traumatismes cutanés, l'amélioration du matériel d'éducation des patients et le développement de classes préparatoires de psychoéducation de groupe. Des évaluations quotidiennes consciencieuses de la peau dans l'unité de traitement ainsi qu'une évaluation hebdomadaire lors de l'examen des radiations peuvent améliorer cette expérience pour les patients.

\section{RÉFÉRENCES}

Andersen, E. R., Eilertsen, G., Myklebust, A. M., \& Eriksen, S. (2018). Women's experience of acute skin toxicity following radiation therapy in breast cancer. Journal of Multidisciplinary Healthcare, 11, 139-148.

Bolderston, A., Cashell, A., McQuestion, M., Cardoso, M., Summers, C., \& Harris, R. (2018). Evidence or tradition? A Canadian survey of the management of radiation-induced skin reactions. Journal of Medical Imaging and Radiation Sciences, 49(2), 164-172.

Campbell, I.R., \& Illingworth, M.H. (1992). Can patients wash during radiotherapy to the breast or chest wall? A randomized controlled trial. Clin Oncol ( $R$ Coll Radiol), 4(2), 78-82.

Chan, R. J., Webster, J., Chung, B., Marquart, L., Muhtashimuddin, A., \& Garantziotis, S. (2014). Prevention and treatment of acute radiation-induced skin reactions: A systematic review and metaanalysis of randomized controlled trials. BMC Cancer, 14, 53. http://ww.biomedcentral.com/1471-2407/14/53.

Feight, D., Baney, T., Bruce, S., \& McQuestion, M. (2011). Putting evidence into practice: Evidence-based interventions for radiation dermatitis; Clin J Oncol Nurs, 15(5), 481-492.

Ferreira, E. B., Vasques, C. I., Gadia, R., Chan, R. J., Guerra, E. N. S., Mezzomo, L. A., Canto, G. D. L., Diniz, P. E., \& Reis, D. (2017). Topical interventions to prevent acute radiation dermatitis in head and neck cancer patients: A systematic review. Supportive Care in Cancer, 25(3), 1001-1011.

Gillan, C., Di Prospero, L., Harnett, N., \& Holden, L. (2014). Research for the Radiation therapist; From question to culture. Apple Academic Press

Glover, D., \& Harmer, V. (2014). Radiotherapy induced skin reactions: Assessment and management. British Journal of Nursing, 23(Suppl. 2), S28-S35.

Guba, E., \& Lincoln, Y. (1985). Naturalistic Inquiry. Sage.
La pratique actuelle ne limite plus le calendrier d'application des produits (Morley et al., 2014; Tse et al., 2016). Les patients sont désormais encouragés à nettoyer la peau intacte avec de l'eau et du savon et à utiliser des lotions, des crèmes, des pommades et des pansements sur leur peau avant le traitement. Ils ne souffrent donc plus autant d'inconforts, de douleurs et de tiraillements de la peau comme quand on leur disait d'éviter tout produit pour une période arbitraire avant le traitement (Morley et al., 2013; Tse et al., 2016). Même si la nécessité de modifier les pratiques est prouvée, des incohérences subsistent dans les différents milieux de pratique et parmi les cliniciens, ce qui conduit à des informations trompeuses et à un soutien inégal aux patients subissant un traitement par irradiation (Bolderston et al., 2018). Les guides de pratique clinique organisationnels basés sur des analyses de la littérature et l'élaboration de lignes directrices peuvent être efficaces pour modifier les pratiques dans les différents milieux cliniques et améliorer l'expérience et les attentes des patients présentant des réactions cutanées à la suite de leur radiothérapie pour un cancer de la tête et du cou (ONS, 2020). La connaissance par les cliniciens des nouveaux articles, des normes et des pratiques actuelles, ainsi que le fait d'aider les patients et les familles à savoir à quoi s'attendre, auront un effet positif sur l'expérience des patients ayant une réaction cutanée radio-induite pendant et après leur traitement contre un cancer de la tête et du cou.

Harris, R. (1997). Consistency of patient information. Is this happening? Cancer Nursing, 20(4), 274-6.

Haisfield-Wolfe, M.E., Brown, C., Richardson, M., \& Webster, K. (2015). Variations in symptom severity patterns among oropharyngeal and laryngeal cancer outpatients during radiation treatment: A pilot study. Cancer Nursing, 38(4), 279-287.

Laszewski, P., Zelko, C., Andriths, L., Cruz, E. V., Bauer, C., \& Magnan, M. A. (2016). Patient preference for instructional reinforcement regarding prevention of radiation dermatitis. Clin J Oncol Nurs; 20(2), 187-91.

McQuestion, M. (2011). Clinical update: Evidence-based skin care management in radiation therapy. Seminars in Oncology Nursing, 27(2), e1-17.

McQuestion, M., \& Fitch, M. (Fall 2016). Patients' experience of receiving treatment for head and neck cancer: Before, during and after treatment. Canadian Oncology Nursing Journal, 26(4), 325-335.

Molassiotis, A., \& Rogers, M. (2012). Symptom experience and regaining normality in the first year following a diagnosis of head and neck cancer. A qualitative longitudinal study. Palliative $Q T$ Supportive Care, 10(3), 197-204.

Morley, L., Cashell, A., Sperduti, A., McQuestion, M., \& Chow, J. C. L. (2014). Evaluating the relevance of dosimetric considerations to patient instructions regarding skin care during radiation therapy. Journal of Radiotherapy in Practice, 13(3), 294-301.

Morse, J. M. (2015). Critical analysis of strategies for determining rigor in qualitative inquiry. Qualitative Health Research, 25(9), $1212-1222$.

Oncology Nursing Society (2020). Putting evidence into practice (PEP) radiodermatitis. https://www.ons.org/pep/radiodermatitis (Note: Updated Guideline to be published fall 2020 - confirmed by ONS) 
Salvo, N., Barnes, E., van Draanen, J., Stacey, E., Mitera, G., Breen, D., Giotis, A., Czarnota, G., Pang, J., \& De Angelis, C. (2010). Prophylaxis and management of acute radiation-induced skin reactions: A systematic review of the literature. Current Oncology, 17(4), 94-112.

Schnur, J. B., Ouellette, S. C., DiLorenzo, T. A., Green, S., \& Montomery, G. H. (2011). A qualitative analysis of acute skin toxicity among breast cancer radiotherapy patients. PsychoOncolog $\gamma, 20,260-268$.

Schnur, J. B. (2010). Radiotherapy-induced skin changes and quality of life. Lancet, 11 (3).

Singh, M., Alavi, A., Wong, R., Akita, S. (2016). Radiodermatitis: A review of our current understanding. Am J Clin Dermatol, 17(3), 277-292. doi: 10.1007/s40257-016-0186-4.

Sutherland, A. E., Bennett, N. C., \& Herst, P. M. (2017). Psychological stress affects the severity of radiation-induced acute skin reactions in breast cancer patients. European Journal of Cancer Care, 26(6), e12737. |https://doi.org/10.1111/ecc.12737

Thorne, S. (2018). What can qualitative studies offer in a world were evidence drives decision? Asia-Pacific Journal of Oncology Nursing, $5(1), 43-45$.

Thorne, S., Kirkham, R., \& MacDonald-Emes, J. (1997). Focus on qualitative methods. Interpretive description: A noncategorical qualitative alternative for developing nursing knowledge. Research in Nursing \& Health, 20(2), 169-177.

Thorne, S., Stephens, J., \& Truant, T. (2016). Building qualitative study design using nursing's disciplinary epistemology. Journal of Advanced Nursing, 72(2), 451-460.
Tong, A., Sainsbury, P., \& Craig, J. (2007). Consolidated criteria for reporting qualitative research (COREQ): A 32-item checklist for interviews and focus groups. International Journal for Quality in Health Care, 19(6), 349-357.

Tse, K., Morley, L., Cashell., Sperduti, A., McQuestion, M., \& Chow, J. C. L. (2016). Dosimetric impacts on skin toxicity for patients using topical agents and dressings during radiotherapy. Journal of Medical Imaging and Radiation Sciences, 47(1).

VanHoose, L., Black, L. L., Doty, K., Sabata, D., Twumasi-Ankrah, P., Taylor, S., \& Johnson, R. (2015). An analysis of the distress thermometer problem list and distress in patients with cancer. Support Care Cancer, 23(4), 1225-1232.

Wells, M. (1998). The hidden experience of radiotherapy to the head and neck: A qualitative study of patients after completion of treatment. J Adv Nurs, 28(4), 840-848. DOI: 10.1111/j.1365-2648.1998x.00714.x

Wells, M. (2007). A study to evaluate nurse-led on-treatment review for patents undergoing radiotherapy for head and neck cancer. $J$ Clin Nurs, 17(11), 1428-1439.

Wong, R., Bensadoun, R., Boers-Doets, C., Eaby-Sandy, B., \& Lacouture, M. (2013). Clinical practice guidelines for the prevention and treatment of acute and late radiation reactions from the MASCC skin toxicity study group. Support Care Cancer; 21, 2933-2948. 